\title{
Constraints on correlations in multiqubit systems
}

\author{
Nikolai Wyderka, Felix Huber, Otfried Gühne \\ Naturwissenschaftlich-Technische Fakultät, Universität Siegen, Walter-Flex-Straße 3, 57068 Siegen, Germany
}

(Dated: April 10, 2019)

\begin{abstract}
The set of correlations between particles in multipartite quantum systems is larger than those in classical systems. Nevertheless, it is subject to restrictions by the underlying quantum theory. In order to better understand the structure of this set, a possible strategy is to divide all correlations into two components, depending on the question of whether they involve an odd or an even number of particles. For pure multi-qubit states we prove that these two components are inextricably interwoven and often one type of correlations completely determines the other. As an application, we prove that all pure qubit states with an odd number of qubits are uniquely determined among all mixed states by the odd component of the correlations. In addition, our approach leads to invariants under the time evolution with Hamiltonians containing only odd correlations and can simplify entanglement detection.
\end{abstract}

PACS numbers: 03.65.Aa, 03.65.Ta, 03.65.Wj

Introduction. - Correlations in quantum mechanics are stronger than their counterparts in the classical world. This fact is important for many applications in quantum information processing. Taking a closer look, however, the former statement sounds like a truism and one realizes that many insights in quantum theory stem from the fact that quantum mechanical correlations are limited and not arbitrarily strong. For instance, the fact that in Bell experiments quantum correlations do not reach the values admissible by non-signaling theories has led to insightful discussions about underlying physical principles [1, 2]. To give another example, for three or more particles monogamy relations bound the entanglement between different pairs of particles, and their study is essential for the progress of entanglement theory [3 6].

If one considers multiparticle systems, however, not only correlations between pairs of particles are relevant but also those between different sets of particles. Any multi-qubit state can be expressed in terms of tensor products of Pauli matrices via the Bloch decomposition. Different terms act on different sets of qubits, describing the correlations between just this set of particles. Consequently, one may ask whether there are any relations between these components of the total correlations. For instance, for three particles, denoted by $A, B$, and $C$, three different contributions can be distinguished (see also Fig. 1): First, there are single-body terms, acting on individual parties alone and determining the single party density matrices. Second, there are two-body correlations acting on the pairs $A B, B C$, and $C A$. Finally, there are three-body correlations acting on all three particles $A B C$. So the question arises: Are these three contributions independent of each other or is one of them determined by the others?

In this paper we present an approach to answer this and more general questions for multi-qubit systems. We identify two components of the correlations, depending on the question of whether they act on an odd or even

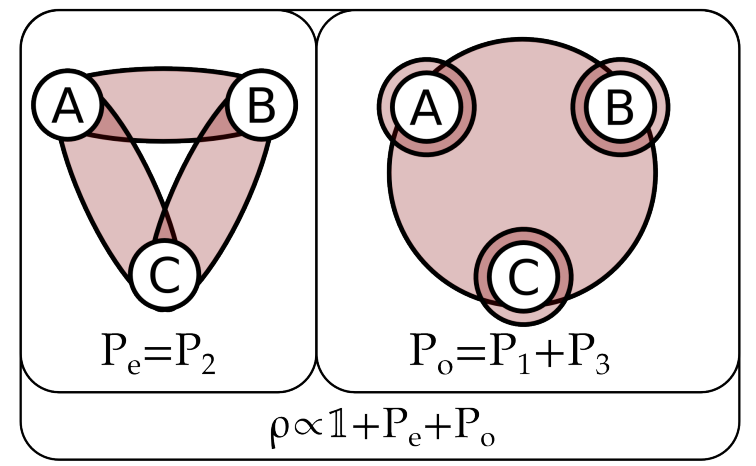

Figure 1. Visualization of the decomposition of a threeparticle state $\rho$ into even and odd correlations. A state $\rho$ is expanded in Bloch representation as $\rho \propto \mathbb{1}+P_{1}+P_{2}+\ldots$, where $P_{j}$ denotes all terms containing $j$-body correlations. We prove that the even correlations $P_{\mathrm{e}}$ are determined by the odd correlations $P_{\mathrm{o}}$ for pure states of an odd number of qubits, so the three qubit state is completely determined by $P_{\mathrm{o}}$.

number of particles. We prove that the even correlations and odd correlations obey strong relations, one component often completely determining the other one. Besides their fundamental interest, our results have several practical applications: We prove that all pure qubit states with an odd number of qubits are uniquely determined among all mixed states by the odd component of the correlations. This generalizes a famous result by Wootters and co-workers for three particles [7]. In addition, our approach can be used to characterize ground states arising from Hamiltonians having even or odd interactions only, and the time evolution under Hamiltonians having the odd component only. Finally, we apply our insights to simplify the task of entanglement detection in certain scenarios.

The Bloch representation. - This representation is obtained by expanding an $n$-qubit quantum state $\rho$ in terms 
of tensor products of Pauli operators. So we can write

$$
\rho=\frac{1}{2^{n}} \sum_{\alpha_{1}, \ldots, \alpha_{n}} c_{\alpha_{1} \ldots \alpha_{n}} \sigma_{\alpha_{1}} \otimes \ldots \otimes \sigma_{\alpha_{n}},
$$

where $\alpha_{i} \in\{0,1,2,3\}, \sigma_{0}=\mathbb{1}$, and $\sigma_{1}, \sigma_{2}, \sigma_{3}$ are the usual Pauli matrices. The coefficients $c_{\alpha_{1} \ldots \alpha_{n}}$ are given by the expectation values $c_{\alpha_{1} \ldots \alpha_{n}}=\operatorname{Tr}\left(\sigma_{\alpha_{1}} \otimes \ldots \otimes \sigma_{\alpha_{n}} \rho\right)$.

In our approach we will sort the terms in the Bloch representation according to the number of qubits they act on. First, we can assign to any basis element $\sigma_{\alpha_{1}} \otimes$ $\ldots \otimes \sigma_{\alpha_{n}}$ its weight, $\operatorname{wt}\left(\sigma_{\alpha_{1}} \otimes \ldots \otimes \sigma_{\alpha_{n}}\right):=\left|\left\{i \mid \alpha_{i} \neq 0\right\}\right|$, as the number of non-trivial Pauli matrices. Then, we group the terms in the decomposition according to their weight

$$
\rho=\frac{1}{2^{n}}\left(\mathbb{1}^{\otimes n}+\sum_{j=1}^{n} P_{j}\right),
$$

where $P_{j}$ denotes the sum over all contributions of weight $j$. We call $P_{j}$ also the $j$-body correlations, being determined by the expectation values taken on groups of $j$ particles. As an example, consider the two-qubit Bell state $\left|\Psi^{+}\right\rangle=(|01\rangle+|10\rangle) / \sqrt{2}$, for which the corresponding density operator reads $\left|\Psi^{+}\right\rangle\left\langle\Psi^{+}\right|=\left(\mathbb{1} \otimes \mathbb{1}+\sigma_{x} \otimes\right.$ $\left.\sigma_{x}+\sigma_{y} \otimes \sigma_{y}-\sigma_{z} \otimes \sigma_{z}\right) / 4$, so we have $P_{1}=0$ and $P_{2}=\sigma_{x} \otimes \sigma_{x}+\sigma_{y} \otimes \sigma_{y}-\sigma_{z} \otimes \sigma_{z}$.

As our main starting point, we further group the operators according to the parity of their weight and define

$$
P_{\mathrm{e}}:=\sum_{j \text { even, } j \neq 0} P_{j}, \quad P_{\mathrm{o}}:=\sum_{j \text { odd }} P_{j} .
$$

Note that $P_{0}=\mathbb{1}^{\otimes n}$ is excluded from $P_{\mathrm{e}}$. Then we can write states in the even-odd-decomposition (see Fig. 1)

$$
\rho=\frac{1}{2^{n}}\left(\mathbb{1}+P_{\mathrm{e}}+P_{\mathrm{o}}\right) .
$$

The central point of our paper is that there are strong relations between $P_{\mathrm{e}}$ and $P_{\mathrm{o}}$, and in many cases one determines the other.

State inversion. - Our approach is based on the state inversion map, which, for any qubit state, can be defined as follows [8, 9]:

$$
\tilde{\rho}:=\sigma_{y}^{\otimes n} \rho^{\mathrm{T}} \sigma_{y}^{\otimes n} .
$$

Physically, the state inversion is obtained by complex conjugation followed by a spin flip. This can be represented by the anti-unitary inversion operator $F:=$ $\left(i \sigma_{y}\right)^{\otimes n} C$ 10. Here, first the complex conjugation $C$ is performed and then $\left(i \sigma_{y}\right)^{\otimes n}$ is applied to a pure state. We have that $F^{\dagger}=(-1)^{n} F$ and for pure states we write $|\tilde{\psi}\rangle=F|\psi\rangle$. It follows that pure states remain pure under the state inversion. Note that on single-qubit Pauli matrices we have $F \sigma_{i} F^{\dagger}=-\sigma_{i}$ for $i \neq 0$. Thus, the action of $F$ in Bloch decomposition is to flip the sign in front of each term that has an odd weight. Starting from Eq. (4), we can also write

$$
\tilde{\rho}=\frac{1}{2^{n}}\left(\mathbb{1}+P_{\mathrm{e}}-P_{\mathrm{o}}\right) .
$$

This allows for an easier representation of the even and odd correlations, namely,

$$
\mathbb{1}+P_{\mathrm{e}}=2^{n-1}(\rho+\tilde{\rho}), \quad P_{\mathrm{o}}=2^{n-1}(\rho-\tilde{\rho}) .
$$

The key observation is that under the state inversion, pure states of an odd number of qubits are mapped to orthogonal states. This fact was known before [11-15], however, we give a proof that allows for generalization to qudit systems, for which the statement is new.

Observation 1. For pure n-qudit states $\rho=|\psi\rangle\langle\psi|$ with $n$ odd we have that

$$
\rho \tilde{\rho}=0 .
$$

Proof. Let $\rho=|\psi\rangle\langle\psi|$ be the pure quantum state and denote the $n$ parties by $A_{1}, \ldots, A_{n}$. Using the Schmidt decomposition one can verify that for any bipartition $M \mid \bar{M}$ of the parties one has for the reduced state $\rho_{M}:=\operatorname{Tr}_{\bar{M}}(\rho)$ the relation

$$
\left(\rho_{M} \otimes \mathbb{1}_{\bar{M}}\right) \rho=\left(\rho_{\bar{M}} \otimes \mathbb{1}_{M}\right) \rho .
$$

Let us denote by $\rho_{i j \ldots}=\rho_{\left\{A_{i}, A_{j}, \ldots\right\}} \otimes \mathbb{1}$ the reduced state of parties $A_{i}, A_{j}, \ldots$, padded by identities which are acting trivially on the other particles. Then, state inversion can be written as a sum over its reductions 16 .

$$
\tilde{\rho}=\mathbb{1}-\sum_{1 \leq i \leq n} \rho_{i}+\sum_{1 \leq i<j \leq n} \rho_{i j}-\ldots \pm \rho .
$$

Note that complementary reductions have the opposite sign since $n$ is odd. Thus, multiplying this equation with $\rho$ and using Eq. (9), every term cancels one of the others and we have $\rho \tilde{\rho}=0$.

In the qudit case, pure states do not stay pure under the state inversion, but are mapped to positive operators. This generalization is studied in a later section.

Results for an odd number of qubits. - Throughout this section, we consider pure qubit states of an odd number of parties, denoted by $\left|\psi^{\text {odd }}\right\rangle$. We can directly prove our first main result.

Observation 2. For pure n-qubit states $\left|\psi^{\text {odd }}\right\rangle$, written in the even-odd decomposition as in Eq. (4), we have that (1) the even and odd components of the correlations commute: $\left[P_{\mathrm{e}}, P_{\mathrm{o}}\right]=0$;

(2) the odd correlations uniquely determine the even correlations via

$$
\mathbb{1}+P_{\mathrm{e}}=\frac{1}{2^{n-1}} P_{\mathrm{o}}^{2} ;
$$

(3) the eigenvalues $\Lambda=\left(\lambda_{1}, \ldots, \lambda_{2^{n}}\right)$ of $P_{\mathrm{e}}$ and $P_{\mathrm{o}}$ are

$$
\begin{aligned}
& \Lambda\left(P_{\mathrm{e}}\right)=\left(2^{n-1}-1,2^{n-1}-1,-1, \ldots,-1\right), \\
& \Lambda\left(P_{\mathrm{o}}\right)=\left(2^{n-1},-2^{n-1}, 0, \ldots, 0\right) .
\end{aligned}
$$


Proof. We use Eq. (7) to write

$$
\begin{aligned}
P_{\mathrm{o}} & =2^{n-1}\left(\left|\psi^{\text {odd }}\right\rangle\left\langle\psi^{\text {odd }}|-| \tilde{\psi}^{\text {odd }}\right\rangle\left\langle\tilde{\psi}^{\text {odd }}\right|\right), \\
\mathbb{1}+P_{\mathrm{e}} & =2^{n-1}\left(\left|\psi^{\text {odd }}\right\rangle\left\langle\psi^{\text {odd }}|+| \tilde{\psi}^{\text {odd }}\right\rangle\left\langle\tilde{\psi}^{\text {odd }}\right|\right) .
\end{aligned}
$$

From Observation 1 it follows that both $\mathbb{1}+P_{\mathrm{e}}$ and $P_{\mathrm{o}}$ are diagonal in the same basis and commute. The eigenvalues then can be read off. Furthermore, Eq. (11) can be directly verified in the common eigenbasis.

The fact that $P_{\mathrm{e}}$ is given by $P_{\mathrm{o}}$ for pure states can be restated in the language of uniqueness: Pure qubit states of an odd number of parties are uniquely determined among pure states (UDP) by the odd correlations. This leads to the converse question of whether these states are also determined by the even correlations $P_{\mathrm{e}}$. The answer to this question is negative, but the set of compatible states is rather small.

Remark 3. Given the even correlations $P_{\mathrm{e}}$ of a pure $n$ qubit state $\left|\psi^{\text {odd }}\right\rangle$, the set of admissible odd correlations $P_{\mathrm{o}}$ to retrieve a pure state again is a two-parameter family. The proof is given in Appendix A.

Application I: Uniqueness among all states. - So far, we have shown that for an odd number of parties, the odd correlations uniquely determine the state among all pure states. This is already a generalization of previous results 17, 18, but one can ask the more general question, whether a state is determined by the correlations among all states (UDA), pure or mixed [19. For that question, some results are known [7, 20, which we can generalize now.

Corollary 4. Consider a pure qubit state $|\psi\rangle$ of $n$ parties where $n$ is odd. Then the state is uniquely determined among all mixed states by $P_{\mathrm{o}}$.

Proof. Recall that in the even-odd decomposition, the state reads $|\psi\rangle\langle\psi|=\left(\mathbb{1}+P_{\mathrm{e}}+P_{\mathrm{o}}\right) / 2^{n}$. Suppose there were a mixed state $\rho$ with the same odd correlations. Then we could write it as a convex sum of pure states,

$$
\rho=\sum_{i} p_{i} \frac{1}{2^{n}}\left(\mathbb{1}+P_{\mathrm{e}}^{(i)}+P_{\mathrm{o}}^{(i)}\right),
$$

where $\sum_{i} p_{i}=1$ and $\sum_{i} p_{i} P_{\mathrm{o}}^{(i)}=P_{\mathrm{o}}$. From Observation 2 we know that $P_{\mathrm{o}}$ has two non-vanishing eigenvalues $\lambda_{o_{ \pm}}= \pm 2^{n-1}$, and the same holds for every $P_{\mathrm{o}}^{(i)}$ as they originate from pure states. Because the largest eigenvalue of the sum equals the sum of all the maximal eigenvalues, all $P_{\mathrm{o}}^{i}$ must share the same corresponding eigenvector. The same is true for the second and lowest eigenvalue. Thus, $P_{\mathrm{o}}^{(i)}=P_{\mathrm{o}}^{(j)}$ for all $i, j$ follows. As the $P_{\mathrm{e}}^{(i)}$ are uniquely determined by the $P_{\mathrm{o}}^{(i)}$, they also coincide and therefore $\rho=|\psi\rangle\langle\psi|$.

This result can be seen as a generalization of Ref. [7], where it was shown that almost all three-qubit states are determined among all states by $P_{1}$ and $P_{2}$. Corollary 4 shows that all three-qubit states are determined among all states by $P_{1}$ and $P_{3}$, and it is remarkable that this generalizes to all odd numbers of parties.
An immediate consequence of Corollary 4 is that all pure states of an odd number of parties are unique ground states of odd-body Hamiltonians. More precisely, choosing $H=-P_{\mathrm{o}}=2^{n-1}\left(\left|\tilde{\psi}^{\text {odd }}\right\rangle\left\langle\tilde{\psi}^{\text {odd }}|-| \psi^{\text {odd }}\right\rangle\left\langle\psi^{\text {odd }}\right|\right)$ yields a specific example of such a Hamiltonian.

Results for an even number of qubits. - We now turn to the case of even $n$, and throughout this section, $\left|\psi^{\text {even }}\right\rangle$ denotes a pure state on an even number of qubits. Although in this case $|\psi\rangle$ and $|\tilde{\psi}\rangle$ do not need to be perpendicular, one can gain some insight on the even and odd components of the correlations. We denote the overlap by $|\langle\tilde{\psi} \mid \psi\rangle|=\alpha$ with a positive number $\alpha$ such that $\operatorname{Tr}(\rho \tilde{\rho})=\alpha^{2}$.

For pure states and $n=2, \alpha$ is just the concurrence [8]. For pure states with $n \geq 2, \alpha$ is known as the $n$ concurrence of a state and is known to be an entanglement monotone [11. For our purpose, we need to distinguish three cases: The case where $\alpha=0$, the case of $0<\alpha<1$ and that of $\alpha=1$.

If $\alpha=0$, we recover the case of an odd number of qubits and the same results are valid. Examples for such states are the $W$-state, $|W\rangle=(|0 \ldots 01\rangle+\ldots+$ $|10 \ldots 0\rangle) / \sqrt{n}$, and all completely separable states. In this case, all the results from the previous sections apply and $P_{\mathrm{o}}$ determines $P_{\text {e }}$.

If $\alpha=1,|\psi\rangle \propto|\tilde{\psi}\rangle$, which means that there are only even correlations present in $|\psi\rangle$ and $P_{\mathrm{o}}=0$. In this case, the even correlations are not determined by the odd correlations at all. One prominent example for such a state is the $n$-party Greenberger-Horne-Zeilinger (GHZ) state, $|\mathrm{GHZ}\rangle=(|0 \ldots 0\rangle+|1 \ldots 1\rangle) / \sqrt{2}$.

If $0<\alpha<1$, even though the results from the previous chapter do not apply, the spectrum of $P_{\mathrm{e}}$ is still rather fixed, leading to the following:

Observation 6. Let $\left|\psi^{\text {even }}\right\rangle$ be a pure qubit state with $\left|\left\langle\psi^{\text {even }} \mid \tilde{\psi}^{\text {even }}\right\rangle\right|^{2}=\alpha^{2} \neq 0$. Write $\left|\psi^{\text {even }}\right\rangle$ in the evenodd decomposition as in Eq. (4). Then

(1) the even correlations $P_{\mathrm{e}}$ uniquely determine the odd correlations $P_{\mathrm{o}}$ up to a sign; and

(2) the family of pure states having the same odd correlations $P_{\mathrm{o}}$ as $\left|\psi^{\text {even }}\right\rangle$ is one-dimensional. The even correlations can be parametrized in terms of $P_{\mathrm{o}}$.

The proof of this Observation is given in Appendix B. The results of all the previous observations are summarized in Table 1

A statement similar to Corollary 4 is not true for an even number of parties with $\alpha \neq 0$, as the family of mixed states $p \rho+(1-p) \tilde{\rho}=\left[\mathbb{1}+P_{\mathrm{e}}+(2 p-1) P_{\mathrm{o}}\right] / 2^{n}$ shares the same even body correlations, unless $\alpha=1$, in which case $P_{\mathrm{o}}=0$ and the state is determined.

As a final remark, note that pure states mixed with white noise can be reconstructed as well from knowledge of $P_{\mathrm{o}}\left(P_{\mathrm{e}}\right)$ for $n$ odd ( $n$ even), as the noise parameter can be deduced from the eigenvalues of the operators.

Application II: Ground states. - Some of our findings can be related to the Kramers theorem 21. Consider a 


\begin{tabular}{|c||c|c|}
\hline & $n$ even and $0<\alpha<1$ & $n$ odd or $\alpha=0$ \\
\hline \hline$P_{\mathrm{o}}$ given & $\begin{array}{c}\text { One-dimensional } \\
\text { solution space for } P_{\mathrm{e}}\end{array}$ & $\begin{array}{c}P_{\mathrm{e}} \text { is uniquely } \\
\text { determined }\end{array}$ \\
\hline$P_{\mathrm{e}}$ given & $\begin{array}{c} \pm P_{\mathrm{o}} \text { is uniquely de- } \\
\text { termined up to the sign }\end{array}$ & $\begin{array}{c}\text { Two-dimensional } \\
\text { solution space for } P_{\mathrm{o}}\end{array}$ \\
\hline
\end{tabular}

Table I. Summary of the relations between the even and odd components of pure state correlations as derived in Observation 2, Remark 3 and Observation 6. The detailed relations can be found in the corresponding proofs. Additionally, if $n$ is even and $\alpha=1$, the state exhibits only even correlations and given $P_{\mathrm{e}}$, only $P_{\mathrm{o}}=0$ is compatible.

Hamiltonian that contains even-body interactions only, such as the Ising model without external field or the $t$ $J$-model. A unique ground state of such a Hamiltonian must have even correlations only. This, however, is not possible if $n$ is odd, in which case odd correlations must be present according to Eq. (11). On the other hand, if $n$ is even, then the ground state must belong to the class of even states, i.e., $\alpha=1$. Second, consider Hamiltonians with odd-body interactions only. The ground-state energy of such Hamiltonians is a function of $P_{\mathrm{o}}$ only. Thus, a unique ground state for $n$ even can only be a state which is determined uniquely by $P_{\mathrm{o}}$, which are exactly the states perpendicular to their inverted states, i.e., having $\alpha=0$ like the $W$-state or product states.

Application III: Unitary time evolution. - Another application concerns the orbits of certain states under the time evolution of Hamiltonians. Here, our approach allows one to re-derive and understand previous results from Ref. [12, where a completely different approach was used. Consider a Hamiltonian $H_{\mathrm{o}}$ consisting of odd-body interactions only. Then, any operator $P$ evolves in time as

$$
P(t)=e^{-i H_{\mathrm{o}} t} P e^{i H_{\mathrm{o}} t}=\sum_{m=0}^{\infty} \frac{(-i t)^{m}}{m !}\left[H_{o}, P\right]_{m},
$$

where $\left[H_{\mathrm{o}}, P\right]_{m}:=\left[H_{\mathrm{o}},\left[H_{\mathrm{o}}, P\right]_{m-1}\right]$ is the $m$-times nested commutator with $\left[H_{\mathrm{o}}, P\right]_{0}=P$.

Now, recall that we denote by wt $(T)$ the weight of a tensor product of Pauli matrices. For these weights, Lemma 1 from Ref. 22, adapted for the case of commutators, can be used. It states that for the weight of the commutator of two tensor products $S$ and $T$ one has that:

$$
\operatorname{wt}([S, T]) \equiv \operatorname{wt}(S)+\operatorname{wt}(T)+1 \quad(\bmod 2),
$$

provided that the commutator does not vanish. This lemma encodes the commutator rules of the Pauli matrices. Therefore, by linearity, commuting two odd or two even Hermitian operators yields an odd operator, while commuting an even and an odd operator yields an even operator.
Consider, for example, the three-qubit operators $S=$ $\sigma_{x} \otimes \sigma_{y} \otimes \sigma_{z}+\mathbb{1} \otimes \mathbb{1} \otimes \sigma_{y}$ and $T=\mathbb{1} \otimes \sigma_{x} \otimes \sigma_{z}$. Then, $S$ has odd and $T$ has even weight. Their commutator is given by $[S, T]=-2 i \sigma_{x} \otimes \sigma_{z} \otimes \mathbb{1}+2 i \mathbb{1} \otimes \sigma_{x} \otimes \sigma_{x}$, which has even weight.

Thus, if $H$ and $P$ are odd, all the nested commutators in Eq. 15 are odd too, and $P(t)$ stays odd for all times $t$. On the other hand, if $H$ is odd but $P$ is even, then $P(t)$ remains even. By Eqs. (4) and (6), $\tilde{\rho}$ evolves too as $\exp (-i H t) \tilde{\rho} \exp (i H t)$, as the state inversion and the time evolution commute in this case. Therefore, given a quantum state $\rho$, the overlap $\alpha^{2}=\operatorname{Tr}(\rho \tilde{\rho})$ stays constant for all times. This is also true for mixed states. In that case, the result also holds for the $n$-concurrence $C_{n}$, given by the convex roof construction for $\alpha$, as the value of $\operatorname{Tr}(\rho \tilde{\rho})$ stays constant for any decomposition of $\rho$ into a sum over pure states 23 . So we have the following.

Observation 7. Any quantum state $\rho(t)$, whose time evolution is governed by an odd-body interacting Hamiltonian has a constant value of $\alpha$ and $C_{n}$.

This result can be useful as follows: Recent experiments enabled the observation of the spreading of quantum correlations under interacting Hamiltonians for systems out of thermal equilibrium [24, 25]. Observation 7 shows that large classes of Hamiltonians preserve certain properties of a quantum state and deviations thereof may be used to characterize the actually realized Hamiltonian. For instance, Refs. 26, 27] proposed methods to engineer Hamiltonians with three-qubit interactions only. Experimentally, the $n$-concurrence is not easy to measure; however, bounds can be found with simple methods [28, 30]. A simple scheme that detects even-body terms in the Hamiltonian is the following.

Start with any state $|\psi(0)\rangle$ with zero $n$-concurrence and let it evolve under the Hamiltonian in question. After a fixed time $t_{0}$, the state can be decomposed as

$$
\left|\psi\left(t_{0}\right)\right\rangle=\sqrt{F}|\mathrm{GHZ}\rangle+\sqrt{1-F}|\chi\rangle
$$

with $\langle\mathrm{GHZ} \mid \chi\rangle=0$. The $n$-concurrence of the state is given by

$$
\begin{aligned}
C_{n}\left(\left|\psi\left(t_{0}\right)\right\rangle\right)= & \left|\left\langle\psi\left(t_{0}\right) \mid \tilde{\psi}\left(t_{0}\right)\right\rangle\right| \\
= & \mid F\langle\mathrm{GHZ} \mid \mathrm{GHZ}\rangle+(1-F)\left\langle\chi\left|\sigma_{y}^{\otimes n}\right| \chi^{*}\right\rangle \\
& +\sqrt{F(1-F)}\left(\left\langle\mathrm{GHZ}\left|\sigma_{y}^{\otimes n}\right| \chi^{*}\right\rangle+\text { H.c. }\right) \mid \\
= & |F+(1-F)\langle\chi \mid \tilde{\chi}\rangle|,
\end{aligned}
$$

as $\left\langle\mathrm{GHZ}\left|\sigma_{y}^{\otimes n}\right| \chi^{*}\right\rangle=\left\langle\mathrm{GHZ} \mid \chi^{*}\right\rangle=\langle\mathrm{GHZ} \mid \chi\rangle^{*}=0$. The right hand side is always lower bounded by

$$
C_{n}(|\psi(t)\rangle) \geq F-(1-F)
$$

If $F>50 \%$, the concurrence is non-zero and even-body interactions must have been present. Therefore, lowconcurrence states cannot approximate the GHZ state under the time evolution with odd-body Hamiltonians. 
Combining this result with the one about ground states of odd-body Hamiltonians, we arrive at the following

Observation 8. If $n$ is even, it is not possible to produce a GHZ state from a $W$-state (or any state with $C_{n}=0$ ) by unitary or adiabatic time evolution under Hamiltonians with odd interactions only.

Application IV: Entanglement detection. - The results of this paper yield insight into the structure of pure quantum states that is still subject to ongoing research [31.

Consider a pure state of $n$ qubits with $n$ being odd. Suppose that the odd correlations $P_{1}, P_{3}, \ldots, P_{n-2}$ are given. If the state is biseparable, there are $(n-1) / 2$ different possibilities of biseparation: It could be biseparable along a cut between one qubit and the other $n-1$ qubits, or between two qubits and $n-2$, etc., up to $(n-1) / 2$ qubits and $(n+1) / 2$ qubits. The first case can be tested for by checking for each party whether the corresponding one-particle reduced state is pure. This can be done due to knowledge of $P_{1}$. The second case, namely, two qubits vs. $n-2$ qubits can be tested by assuming that the $(n-2)$-qubit state is pure and trying to reconstruct the appropriate even correlations. According to Corollary 4, this is only possible if the state was indeed pure. This procedure can be applied for all other splittings as well. Thus, the information on genuine multipartite entanglement in pure states is embodied in the odd correlations $P_{1}, P_{3}, \ldots, P_{n-2}$ already, where no knowledge of the highest correlations $P_{n}$ is needed. This is in contrast to the case of mixed states.

Possible generalizations.-

While the results obtained in this paper are valid for qubit systems only, some extensions to higherdimensional systems are possible, as we will discuss now. As stated in the main text, the state inversion can be generalized to systems of internal dimension $d$, as discussed in 32,

$$
\tilde{\rho}:=\mathbb{1}^{\otimes n}-\sum_{i=1}^{n} \rho_{(i)}+\sum_{i<j} \rho_{(i j)}-\ldots \pm \rho .
$$

This yields a positive operator [16, 33, which can be normalized to a proper state. In the Bloch decomposition, the inversion reads

$$
\tilde{\rho}=\frac{(d-1)^{n}}{d^{n}} \sum_{j=0}^{n}\left(\frac{1}{1-d}\right)^{j} P_{j} .
$$

However, for $n>1$ and $d>2$ pure states do not stay pure under the state inversion. Thus, the state inversion cannot be represented as an operator acting on vectors in Hilbert space anymore, but only as a channel. Nevertheless, this generalization has recently been used to gain insight into the distribution of entanglement in higher dimensional many-body systems 32 .

Another generalization concerns the nature of the inversion operator. Instead of flipping the sign of all nontrivial Pauli operators, one can generalize this to only flipping certain ones. The most general form of such an operator acting on a single qubit reads

$$
F_{\alpha}=i C\left(i \alpha_{0} \mathbb{1}+\sum_{i=1}^{3} \alpha_{i} \sigma_{i}\right)
$$

where the four dimensional vector $\vec{\alpha}$ is normalized. The choice $\alpha=(0,0,1,0)^{\mathrm{T}}$ corresponds to the flip considered above (the signs of all Pauli matrices are flipped), whereas $\alpha=(1,0,0,0)^{\mathrm{T}}$ flips just $\sigma_{y}$ (which corresponds to a transposition of the state), $\alpha=(0,1,0,0)^{\mathrm{T}}$ flips $\sigma_{z}$, and $\alpha=(0,0,0,1)^{\mathrm{T}}$ flips $\sigma_{x}$. Other values of $\vec{\alpha}$ correspond to superpositions of these flips. Indeed, $F_{\alpha}|\psi\rangle$ is a pure state again. For example, setting $\alpha=(0,0,0,1)^{\mathrm{T}}$ allows for a decomposition of states by the number of $\sigma_{x}$ appearing in each term, thus, $P_{\mathrm{e}}$ would consist of all terms with an even number of $\sigma_{x}$. Using this decomposition, analogous results can be derived with similar uniqueness properties.

Discussion. - We introduced the decomposition of multipartite qubit states in terms of even and odd correlations. For pure states, we showed that the even and odd correlations are deeply connected, and often one type of correlations determines the other. This allowed us to prove several applications, ranging from the unique determination of a state by its odd correlations to invariants under Hamiltonian time evolution and entanglement detection.

For future work, it would be highly desirable to generalize the approach to higher-dimensional systems. Some facts about state inversion are collected in the previous section, but developing a general theory seems challenging. Furthermore, it may be very useful if one can extend our theory to a quantitative theory, where the correlations within some subset of particles are measured with some correlation measure and then monogamy relations between the different types of correlations are developed.

Note added. - In a previous version of this manuscript, we claimed that "[...] if a state $|\psi\rangle$ is uniquely determined among all states by certain sets of correlations (for example, odd-body correlations), then $|\psi\rangle$ is the unique ground state of some Hamiltonian having interaction terms from that set only." This statement is not correct, as a recently found analytical counterexample on six qubits shows 34 .

The error in the reasoning occured after Lemma 5: The conclusion "As $\mathcal{R}(|\psi\rangle\langle\psi|)$ is extremal, there exists a linear witness $L$ in the projected space $\mathcal{R}(\mathcal{O})$ of all Hermitian operators $\mathcal{O}$ with $\langle\psi|L| \psi\rangle$ being minimal" is wrong. An explanation of the fallacy is already given in Ref. 35] (cf. Fig. 1): an explicit two-dimensional set is constructed, in which some extremal points can not be separated from all other extremal points by any linear witness. Thus, any such a witness cannot detect a single state uniquely. In our context, such a witness cannot be used as a Hamiltonian with non-degenerate ground state 
space.

We removed the wrong statement and the now unnecessary Lemma 5, and inserted a correct argument for the claim that "[...] all pure states of an odd number of parties are unique ground states of odd-body Hamiltonians."

Acknowledgments. - We thank Jens Siewert, Gavin Brennen and Lorenza Viola for fruitful discussions. This work was supported by the Swiss National Science Foundation (Doctoral Mobility Grant No. 165024), the DFG, the ERC (Consolidator Grant No. 683107/TempoQ), and the House of Young Talents Siegen.

\section{APPENDIX}

\section{A: Proof of Remark 3}

Remark 3. Given the even correlations $P_{\mathrm{e}}$ of a pure $n$ qubit state $\left|\psi^{\text {odd }}\right\rangle$, the set of admissible odd correlations $P_{\mathrm{o}}$ to retrieve a pure state again is a two-parameter family.

Proof. Let $\rho=\left|\psi^{\text {odd }}\right\rangle\left\langle\psi^{\text {odd }}\right|$ and $\tilde{\rho}=\left|\tilde{\psi}^{\text {odd }}\right\rangle\left\langle\tilde{\psi}^{\text {odd }}\right|$, and write $\mathbb{1}+P_{\mathrm{e}}=2^{n-1}(\rho+\tilde{\rho})$. Thus, the eigenvectors with eigenvalue $2^{n-1}$ of $\mathbb{1}+P_{\mathrm{e}}$ are a superposition of $\left|\psi^{\text {odd }}\right\rangle$ and $\left|\tilde{\psi}^{\text {odd }}\right\rangle$. Given only $P_{\mathrm{e}}$, one can choose any of its eigenvectors $|\eta\rangle$ from the two-dimensional subspace of eigenvalue $2^{n-1}-1$. As $|\tilde{\eta}\rangle$ is orthogonal to $|\eta\rangle$, it follows that $\mathbb{1}+P_{\mathrm{e}}=2^{n-1}(|\eta\rangle\langle\eta|+| \tilde{\eta}\rangle\langle\tilde{\eta}|)$. Therefore, every choice of an eigenvector gives rise to compatible correlations $P_{\mathrm{o}}^{(r)}$ via

$$
P_{\mathrm{o}}^{(r)}=2^{n-1}(|\eta\rangle\langle\eta|-| \tilde{\eta}\rangle\langle\tilde{\eta}|),
$$

resulting in the total state $\rho=|\eta\rangle\langle\eta|$. By fixing one of the eigenstates $|\eta\rangle$, one can parametrize all valid solutions by

$$
\begin{aligned}
P_{\mathrm{o}}^{(r)}(\theta, \phi)=2^{n-1}[\cos \theta(|\eta\rangle\langle\eta|-| \tilde{\eta}\rangle\langle\tilde{\eta}|) \\
\left.+\sin \theta\left(e^{i \phi}|\tilde{\eta}\rangle\left\langle\eta\left|+e^{-i \phi}\right| \eta\right\rangle\langle\tilde{\eta}|\right)\right]
\end{aligned}
$$

for all real valued $\theta$ and $\phi$.

\section{B: Proof of Observation 6}

Observation 6. Let $\left|\psi^{\text {even }}\right\rangle$ be a pure qubit state with $|\langle\psi \mid \tilde{\psi}\rangle|^{2}=\alpha^{2} \neq 0$. Write $\left|\psi^{\text {even }}\right\rangle$ in the even-odd decomposition as in Eq. (4). Then

(1) the even correlations $P_{\mathrm{e}}$ uniquely determine the odd correlations $P_{\mathrm{o}}$ up to a sign; and

(2) the family of pure states having the same odd correlations $P_{\mathrm{o}}$ as $\left|\psi^{\text {even }}\right\rangle$ is one-dimensional. The even correlations can be parametrized in terms of $P_{\mathrm{o}}$.

Proof. Let $\rho=\left|\psi^{\text {even }}\right\rangle\left\langle\psi^{\text {even }}\right|$. Before proving the statements, we investigate the eigenvectors and eigenvalues of $P_{\mathrm{e}}$ and $P_{\mathrm{o}}$. As $\mathbb{1}+P_{\mathrm{e}}=2^{n-1}(\rho+\tilde{\rho})$, it must be of rank two if $\alpha \neq 1$. Thus, it has two non-vanishing eigenvalues, lying in the span of $|\psi\rangle$ and $|\tilde{\psi}\rangle$. Calculating

$$
\begin{aligned}
& \left(\mathbb{1}+P_{\mathrm{e}}\right)|\psi\rangle=2^{n-1}\left(|\psi\rangle+\alpha e^{i \phi}|\tilde{\psi}\rangle\right), \\
& \left(\mathbb{1}+P_{\mathrm{e}}\right)|\tilde{\psi}\rangle=2^{n-1}\left(|\tilde{\psi}\rangle+\alpha e^{-i \phi}|\psi\rangle\right)
\end{aligned}
$$

yields the two non-vanishing eigenvalues

$$
1+\lambda_{\mathrm{e}_{ \pm}}=2^{n-1}(1 \pm \alpha)
$$

and the corresponding orthonormal eigenvectors

$$
\left|e_{ \pm}\right\rangle=\frac{1}{\sqrt{2(1 \pm \alpha)}}\left(|\psi\rangle \pm e^{i \phi}|\tilde{\psi}\rangle\right) .
$$

We can also determine the action of $P_{\mathrm{o}}$ on these eigenvectors, which reveals that it is purely off-diagonal in the eigenbasis of $P_{\mathrm{e}}$,

$$
P_{\mathrm{o}}\left|e_{ \pm}\right\rangle=2^{n-1}(\rho-\tilde{\rho})\left|e_{ \pm}\right\rangle=2^{n-1} \sqrt{1-\alpha^{2}}\left|e_{\mp}\right\rangle .
$$

Thus, the eigenvectors of $P_{\mathrm{o}}$ are given by

$$
\left|o_{ \pm}\right\rangle=\frac{1}{\sqrt{2}}\left(\left|e_{+}\right\rangle \pm\left|e_{-}\right\rangle\right)
$$

and the eigenvalues are given by

$$
\lambda_{\mathrm{o}_{ \pm}}= \pm 2^{n-1} \sqrt{1-\alpha^{2}} .
$$

We are now in position to prove the claims. Let us prove statement two first:

(2) By assumption, $P_{\mathrm{o}}$ is known. The eigenvalues determine the overlap $\alpha$ by Eq. (30). Knowledge of $\alpha$ fixes the eigenvalues of any admissible reconstructed $P_{\mathrm{e}}^{(r)}$. The admissible eigenvectors of $P_{\mathrm{e}}^{(r)}$ can be obtained from Eq. (29) to read

$$
\left|e_{ \pm}\right\rangle=\frac{1}{\sqrt{2}}\left(\left|o_{+}\right\rangle \pm\left|o_{-}\right\rangle\right)
$$

However, the eigenvectors $\left|o_{ \pm}\right\rangle$are only unique up to a phase. Taking into account this extra phase while omitting a global phase yields

$$
\left|e_{ \pm}\right\rangle=\frac{1}{\sqrt{2}}\left(\left|o_{+}\right\rangle \pm e^{i \varphi}\left|o_{-}\right\rangle\right) .
$$

This allows us to write all compatible even correlations as

$$
\begin{aligned}
\mathbb{1}+P_{\mathrm{e}}^{(r)}= & \left(1+\lambda_{\mathrm{e}_{+}}\right)\left|e_{+}\right\rangle\left\langle e_{+}\left|+\left(1+\lambda_{\mathrm{e}_{-}}\right)\right| e_{-}\right\rangle\left\langle e_{-}\right| \\
= & 2^{n-1}\left(\left|o_{+}\right\rangle\left\langle o_{+}\left|+\alpha e^{-i \varphi}\right| o_{+}\right\rangle\left\langle o_{-}\right|\right. \\
& \left.\quad+\left|o_{-}\right\rangle\left\langle o_{-}\left|+\alpha e^{i \varphi}\right| o_{-}\right\rangle\left\langle o_{+}\right|\right) .
\end{aligned}
$$

This is a one-dimensional space of admissible reconstructed even correlations, parametrized by $\varphi$.

We now show the first statement:

(1) Assume that now $P_{\mathrm{e}}$ is given. Can we uniquely reconstruct in the odd correlations $P_{\mathrm{o}}$ from knowledge of $P_{\mathrm{e}}$ ? Unfortunately, the eigenvectors $\left|e_{ \pm}\right\rangle$are again only 
determined up to a phase. Therefore, every reconstructed operator $P_{o}^{(r)}$ of the form

$$
\begin{aligned}
P_{\mathrm{o}}^{(r)} & =\lambda_{o_{+}}\left|o_{+}\right\rangle\left\langle o_{+}\left|+\lambda_{-}\right| o_{-}\right\rangle\left\langle o_{-}\right| \\
& =\lambda_{o_{+}}\left(e^{i \varphi}\left|e_{+}\right\rangle\left\langle e_{-}\left|+e^{-i \varphi}\right| e_{-}\right\rangle\left\langle e_{+}\right|\right)
\end{aligned}
$$

for all $\varphi \in \mathbb{R}$ would be a valid operator, such that

$$
\frac{1}{2^{n}}\left(\mathbb{1}+P_{\mathrm{e}}+P_{\mathrm{o}}^{r}\right)
$$

is a pure state again. However, only certain choices of $\varphi$ recreate a $P_{\mathrm{o}}$ which exhibits solely odd correlation in Bloch decomposition. This can be seen as follows: As shown in Lemma 9 below, $\left|e_{ \pm}\right\rangle\left\langle e_{ \pm}\right|$can only exhibit even correlations. This means that $\left|e_{ \pm}\right\rangle$are eigenvectors of the inversion operator $F$ introduced above, i.e. $F\left|e_{ \pm}\right\rangle \propto\left|e_{ \pm}\right\rangle$. Recall that for $n$ even, $F^{\dagger}=F$. Thus, $F\left|e_{+}\right\rangle\left\langle e_{-}\left|F^{\dagger}=e^{i \Lambda}\right| e_{+}\right\rangle\left\langle e_{-}\right|$for some $\Lambda$. The condition that $P_{\mathrm{o}}^{(r)}$ contains only odd correlations can be written as

$$
P_{\mathrm{o}}^{(r)}+\tilde{P}_{\mathrm{o}}^{(r)}=P_{\mathrm{o}}^{(r)}+F P_{\mathrm{o}}^{(r)} F^{\dagger}=0 .
$$

Eq. 35 translates this to

$$
e^{i \varphi}+e^{-i(\varphi-\Lambda)}=0
$$

which exhibits exactly two solutions for $\varphi$. Thus, there are only two possible reconstructions $P_{\mathrm{o}}^{(r)}$, corresponding to the original $P_{\mathrm{o}}$ and its negation, $-P_{\mathrm{o}}$.

All that is left is to show the used assumption that the eigenvectors $\left|e_{ \pm}\right\rangle$exhibit only even correlations. Note, that this is a special case of Kramers theorem [21, stating that the eigenstates of a Hamiltonian exhibiting even correlations only is either at least two-fold degenerate or exhibits itself only even correlations.

Lemma 9. Let $P=\lambda_{+}\left|p_{+}\right\rangle\left\langle p_{+}\left|+\lambda_{-}\right| p_{-}\right\rangle\left\langle p_{-}\right|$be a Hermitian operator which exhibits only even correlations in the Bloch decomposition, $\left\langle p_{+} \mid p_{-}\right\rangle=0$ and $\lambda_{-}<\lambda_{+}$. Then $\left|p_{+}\right\rangle\left\langle p_{+}\right|$and $\left|p_{-}\right\rangle\left\langle p_{-}\right|$also exhibit only even correlations.

Proof. We regard $P$ as a Hamiltonian with the unique ground state $\left|p_{-}\right\rangle$. As $P$ has even correlations only, $F P F^{\dagger}=P$. Thus

$$
\begin{aligned}
\lambda_{-} & =\operatorname{Tr}\left(P\left|p_{-}\right\rangle\left\langle p_{-}\right|\right)=\operatorname{Tr}\left(F P F^{\dagger}\left|p_{-}\right\rangle\left\langle p_{-}\right|\right) \\
& =\operatorname{Tr}\left(P F\left|p_{-}\right\rangle\left\langle p_{-}\right| F^{\dagger}\right),
\end{aligned}
$$

as $F^{\dagger}=F$ if $n$ is even. Thus, also $F\left|p_{-}\right\rangle$is a ground state of $P$. As by assumption the ground state is unique, $F\left|p_{-}\right\rangle \propto\left|p_{-}\right\rangle$must hold true and therefore, $\left|p_{-}\right\rangle\left\langle p_{-}\right|$ exhibits only even correlations. This implies that also $\left|p_{+}\right\rangle\left\langle p_{+}\right|$has even correlations only.

[1] M. Pawłowski, T. Paterek, D. Kaszlikowski, V. Scarani, A. Winter, and M. Zukowski, Nature (London) 461, 1101 (2009).
[2] M. Navascués, Y. Guryanova, M. J. Hoban, and A. Acín, Nature Commun. 6, 6288 (2015).

[3] V. Coffman, J. Kundu, and W. K. Wootters, Phys. Rev. A 61052306 (2000).

[4] T.J. Osborne, and F. Verstraete, Phys. Rev. Lett. 96, 220503 (2006).

[5] C. Eltschka, and J. Siewert, Phys. Rev. Lett. 114, 140402 (2015).

[6] P. Kurzynski, T. Paterek, R. Ramanathan, W. Laskowski, and D. Kaszlikowski, Phys. Rev. Lett. 106, 180402 (2011).

[7] N. Linden, S. Popescu, and W. K. Wootters, Phys. Rev. Lett. 89, 207901 (2002).

[8] W. K. Wootters, Phys. Rev. Lett. 80, 2245 (1998).

[9] S. Hill, and W. K. Wootters, Phys. Rev. Lett. 78, 5022 (1997).

[10] V. Bužek, M. Hillery, and R. F. Werner, Phys. Rev. A 60, R2626 (1999).

[11] A. Wong, and N. Christensen, Phys. Rev. A 63, 044301 (2001).

[12] S. S. Bullock, G. K. Brennen, and D. P. O'Leary, J. Math. Phys. 46, 062104 (2005).

[13] A. Osterloh, and J. Siewert, Phys. Rev. A 72, 012337 (2005).

[14] P. Butterley, A. Sudbery, and J. Szulc, Found. Phys. 36, 83 (2006).

[15] S. Designolle, O. Giraud, and J. Martin, Phys. Rev. A 96, 032322 (2017).

[16] W. Hall, Phys. Rev. A 72, 022311 (2005).

[17] L. Diósi, Phys. Rev. A 70, 010302 (2004).

[18] N. Wyderka, F. Huber, and O. Gühne, Phys. Rev. A 96, 010102 (2017).

[19] J. Chen, H. Dawkins, Z. Ji, N. Johnston, D. Kribs, F. Shultz, and B. Zeng, Phys. Rev. A 88, 012109 (2013).

[20] N. S. Jones, and N. Linden, Phys. Rev. A 71, 012324 (2005).

[21] H. A. Kramers, Proc. R. Acad. Sci. Amsterdam 33, 959 (1930).

[22] F. Huber, O. Gühne, and J. Siewert, Phys. Rev. Lett. 118, 200502 (2017).

[23] A. Uhlmann, Phys. Rev. A 62, 032307 (2000).

[24] M. Cheneau, P. Barmettler, D. Poletti, M. Endres, P. Schauß, T. Fukuhara, C. Gross, I. Bloch, C. Kollath, and S. Kuhr, Nature (London) 481, 484 (2012).

[25] P. Jurcevic, B. P. Lanyon, P. Hauke, C. Hempel, P. Zoller, R. Blatt, and C. F. Roos, Nature (London) 511, 202 (2014).

[26] J. K. Pachos, and E. Rico, Phys. Rev. A 70, 053620 (2004).

[27] H.P. Büchler, A. Micheli, and P. Zoller, Nature Physics 3, 726 (2007).

[28] C. Schmid, N. Kiesel, W. Wieczorek, H. Weinfurter, F. Mintert, and A. Buchleitner, Phys. Rev. Lett. 101, 260505 (2008).

[29] S. J. van Enk, Phys. Rev. Lett. 102, 190503 (2009).

[30] C. Zhang, S. Yu, Q. Chen, H. Yuan, and C. H. Oh, Phys. Rev. A 94, 042325 (2016).

[31] D. Goyeneche, G. Cañas, S. Etcheverry, E. S. Gómez, G. B. Xavier, G. Lima, and A. Delgado, Phys. Rev. Lett. 115, 090401 (2015).

[32] C. Eltschka, and J. Siewert, Quantum 2, 64 (2018).

[33] P. Rungta, V. Bužek, C. M. Caves, M. Hillery, and G. J. Milburn, Phys. Rev. A 64, 042315 (2001).

[34] S. Karuvade, P.D. Johnson, F. Ticozzi, L. Viola, arXiv 
preprint arXiv:1902.09481 (2019).
[35] J. Chen, Z. Ji, B. Zeng, D.L. Zhou, Phys. Rev. A 86, 022339 (2012). 